OPEN

SUBJECT AREAS:

IMAGING

BIOPHYSICS

BIOPHOTONICS

APPLIED PHYSICS

Received

15 June 2011

Accepted

25 October 2011

Published

9 November 2011

Correspondence and requests for materials should be addressed to

P.P.M. (partha@isu. iisc.ernet.in)

\section{Simultaneous multilayer scanning and detection for multiphoton fluorescence} microscopy

\author{
Partha Pratim Mondal' \& Alberto Diaspro ${ }^{2}$
}

\begin{abstract}
${ }^{1}$ Nanobioimaging Laboratory, Department of Instrumentation and Applied Physics, Indian Institute of Science, Bangalore 560012, India, ${ }^{2}$ Nanophysics, Italian Institute of Technology, 16163 Genova, Italy.
\end{abstract}

Fast three-dimensional (3D) imaging requires parallel optical slicing of a specimen with an efficient detection scheme. The generation of multiple localized dot-like excitation structures solves the problem of simultaneous slicing multiple specimen layers, but an efficient detection scheme is necessary. Confocal theta detection (detection at $90^{\circ}$ to the optical axis) provides a suitable detection platform that is capable of cross-talk-free fluorescence detection from each nanodot (axial dimension $\approx 150 \mathrm{~nm}$ ). Additionally, this technique has the unique feature of imaging a specimen at a large working distance with super-resolution capabilities. Polarization studies show distinct field structures for fixed and fluid samples, indicating a non-negligible field-dipole interaction. The realization of the proposed imaging technique will advance and diversify multiphoton fluorescence microscopy for numerous applications in nanobioimaging and optical engineering.

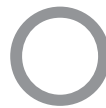

ptical sectioning ${ }^{1}$ and point-by-point scanning are required for most existing fluorescence microscopy techniques. This technique have the advantage of being non-destructive, and they offer the flexibility of slicing along the desired sample layer. Confocal laser scanning microscopy and multiphoton fluorescence microscopy are routinely used to make optical scans of a sample and stack them for 3D reconstruction of biological structures ${ }^{2,3,4,5}$. Selective plane illumination microscopy (SPIM) is a similar technique in which single planes are illuminated sequentially to obtain sectional images ${ }^{7}$. Recently, there has been growing interest in multiphoton super-resolution scanning techniques ${ }^{8,9,10}$. Despite the rapid advances in light microscopy, simultaneous visualization of multiple layers (that includes both excitation and detection) has not yet been achieved. Such a capability would have numerous applications, including, fast imaging (by parallelizing data acquisition), and data correlation across multiple layers of the specimen. For example, simultaneous data from the nucleus and the cell membrane could be recorded and correlated to extract vital information about the internal function of a cell. Furthermore, existing fluorescence imaging techniques offer limited imaging depth-, and poor axial resolution, and they suffer from photobleaching ${ }^{11,12,13,14,15}$. Proposed technique holds the promise of reducing the photobleaching that is prevalent in biological imaging and offers better depth-imaging capability. For this reason, we have previously proposed an excitation technique for obtaining multiple localized field patterns using an optical mask ${ }^{16,17}$. The proposed excitation scheme is straight forward, but the detection scheme is tedious, especially when distinguishing the emission from the nanoscopic dots while simultaneously avoiding cross-talks between them. The goal is to excite multiple specimen layers that are few hundred nanometers apart, and to selectively detect fluorescence from the nanodots almost simultaneously.

In this report, we propose an orthogonal detection scheme for multiphoton multiple excitation spot optical (MMESO) microscopy, in which the emission is collected by an objective that is placed perpendicular to the optical axis. This orthogonal detection was initially developed by Voie et al. ${ }^{1}$ and further developed by Stelzer et al. ${ }^{18}$. It should be noted that, a long working distance objective lens has a low numerical aperture and the converse is also true. For the proposed M-MESO microscopy, confocal theta detection offers an effective solution because this configuration combines the advantage of high resolution and long working distance. Here, we show that, the emission from a single nanodot can be effectively collected without introducing cross-talks from its neighboring nanodots. Orthogonal detection has the following additional features compared to conventional confocal detection: (1) the system axial resolution is effectively the lateral resolution of the detection system, (2) most of the excitation light is cut-off because of the perpendicular detection (and the background light is substantially reduced as a result), and (3) the detection arm is independent of the excitation arm, which provides additional 
flexibility for fluorescence detection without altering the excitation mechanism. We show unique (cross-talk free) identification of an individual nanodot from a series of nanodots by utilizing a confocal theta detection scheme. In principle, simultaneous detection from all of the nanodots can be obtained by introducing a distorted diffraction grating in the detection path of the imaging system ${ }^{19}$, followed by the charge-coupled device (CCD) detector. Interestingly, this detection technique reports nano-particle tracking of approximately $10 \mathrm{~nm}$ along the $z$-axis ${ }^{19}$. Alternately, a $50 / 50$ beam-splitter with an appropriately positioned pinhole can be used in the detection pathway ${ }^{20}$. The proposed imaging technique is a boon to multiphoton microscopy, in which the poor axial resolution of the multiphoton imaging process limits the advantage of thin optical slicing.

\section{Results}

The conventional laser-scanning imaging technique is limited by the classical resolution limit and suffers from slow data acquisition as a result of the point-by-point scan. These shortcomings encourage the development of fast super-resolution fluorescence imaging techniques. Ideally, the technique should have simultaneous multi-layer excitation and cross-talk-free detection for diffraction-unlimited multilayer imaging. These attributes enable several applications, including fast imaging and multi-layer crosscorrelation studies. Here, we study a new imaging system (see Figure 1) and specifically characterize the system point spread function (PSF) under the isotropic emission model with randomly polarized light illumination. Illumination, detection and the system PSF for the proposed $M-$ MESO system are shown in Figure 2. The axial resolution of the proposed system is improved by a factor of approximately 4 over the classical resolution limit, while there is no improvement in the lateral resolution because the perpendicular detection occurs along the lateral axis of the illumination objective. As a result, the axial resolution of the total imaging system is essentially determined by the lateral resolution of the objective $\mathrm{O}_{3}$. Thus, the multi-dot illumination PSF $\left(h_{\text {ill }}\right)$ multiplied by the detection PSF $\left(h_{\text {det }}\right)$ gives the system PSF, which is the desired single-dot (see Figure 2). The corresponding axial and lateral resolutions are approximately, $210 \mathrm{~nm}$ and $150 \mathrm{~nm}$, respectively (see Figure 2). Moreover, a system PSF volume of approximately $27.7 \times 10^{-3}$ femto - lit is obtained. By scanning the objective $\mathrm{O}_{3}$, the fluorescence from all of the nanodots can be recorded, in principle. Moreover, we have carried out studies on the single photon variant of the proposed MESO imaging system. This study facilitates the direct comparison of the single- and multiphoton MESO imaging techniques. The excitation, detection and overall system PSF are shown in Figure 3 for $\alpha_{i l l}=45^{\circ}$ and $\alpha_{\text {det }}=$ $45^{\circ}$. Notably, the density of the nanodots doubles because of the smaller wavelength employed for the single-photon MESO system. The size of the individual nanodot (determined by its full-width half-maximum) is small (lateral resolution $r_{x y}=180 \mathrm{~nm}$ and axial

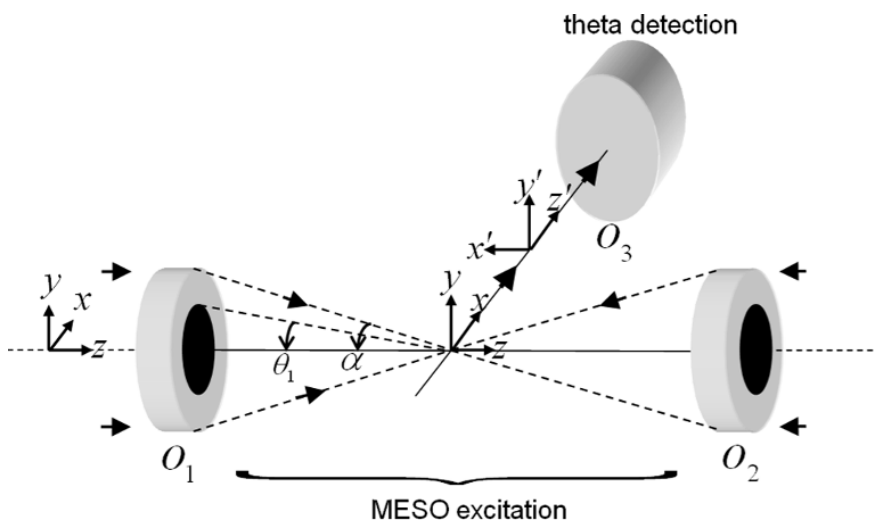

Figure $1 \mid$ Optical configuration of the proposed M-MESO microscopy.

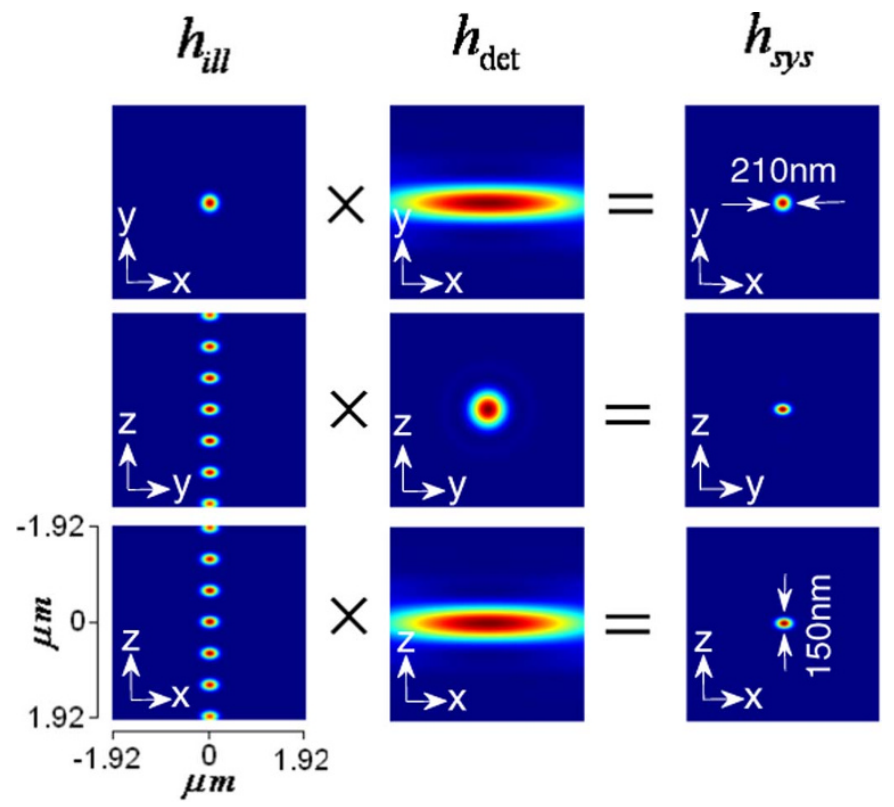

Figure 2 $\mid$ M-MESO system PSF for $\alpha_{i l l}=45^{\circ}$ and $\alpha_{d e t}=30^{\circ}$.

resolution $\Delta z=120 \mathrm{~nm}$ ) for single photon MESO compared to the M-MESO system, for which $r_{x y}=210 \mathrm{~nm}$ and $\Delta z=150 \mathrm{~nm}$. Increased crosstalks have been observed in the single photon variant compared to the multiphoton variant. This increase is evident in Figure 4, which shows the intensity plots obtained along predefined lines passing through the system PSF. These plots indicate prominent sidelobes for single-photon MESO compared to M-MESO, which can be attributed to the doubling of the nanodot density as a result of the shorter excitation wavelength used for single photon MESO. The shorter wavelength modifies the interference condition, thereby altering the distance between nearby nanodots. As a result, the detection PSF picks up fluorescence from the nearby nanodots (both sides). This difference clearly illustrates the advantage of M-MESO over its single photon variant, in addition to the other advantages of the multiphoton excitation process.

In a high resolution multiphoton imaging system, the advantage of axial super-resolution at long working distances is of paramount importance. For this reason, we have investigated the system
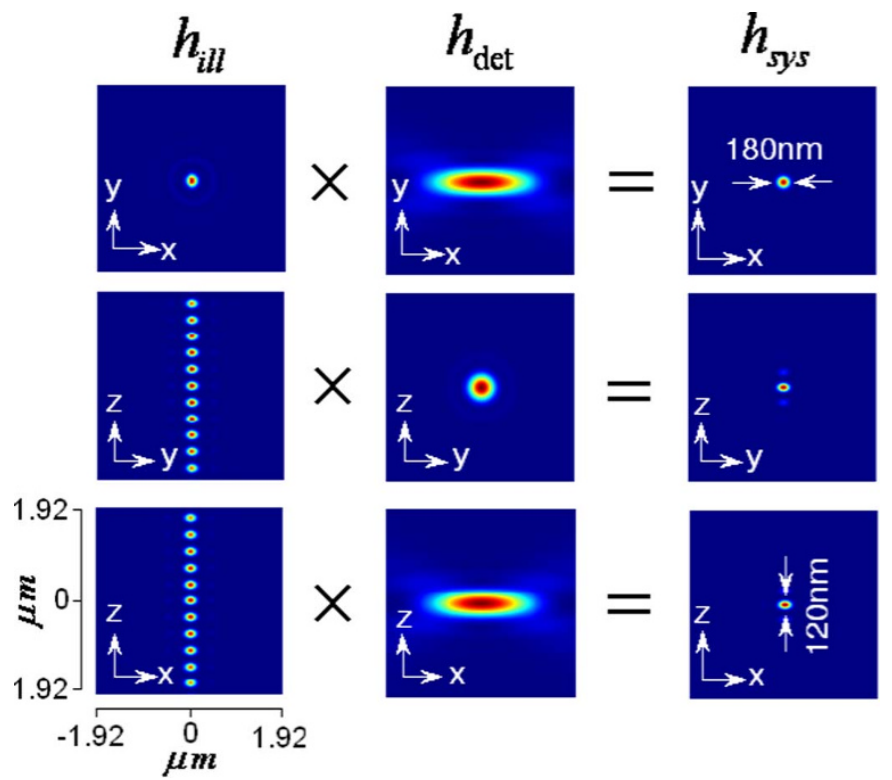

Figure $3 \mid$ Single photon MESO PSF for $\alpha_{i l l}=45^{\circ}$ and $\alpha_{\text {det }}=45^{\circ}$. 


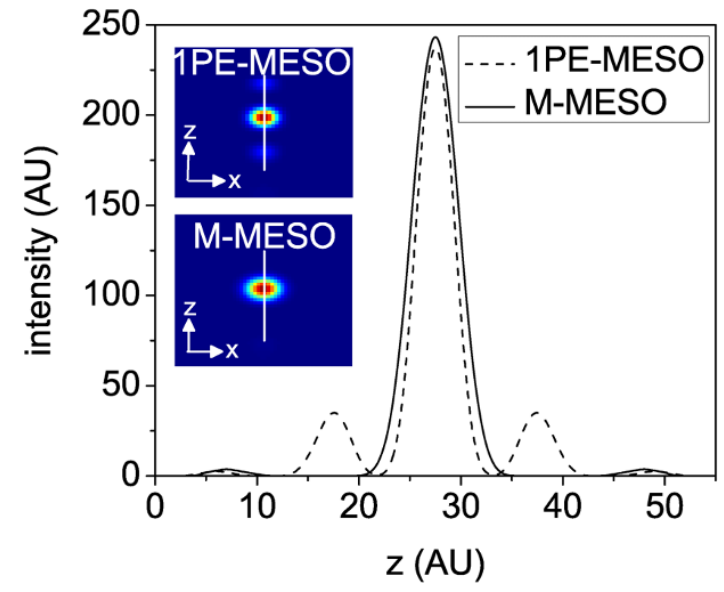

Figure $4 \mid$ Intensity plots along the $X Z$-plane. The inset shows the corresponding single- (1PE) and two-(2PE) photon MESO PSFs.

performance at a small illumination aperture angle $\left(\alpha_{i l l}=18^{\circ}\right)$ and varying detection aperture angles $\left(\alpha_{\text {det }}\right)$. At low numerical aperature of the detection objective $\left(\alpha_{d e t}=30^{\circ}\right)$, a sidelobe appears because of the increased crosstalks from both of the neighboring excitation dots (indicated by red arrows in Figure 5(a)). Further evidence of this cross-talk is shown in the intensity plots (see Figure 5(b)). The effect is substantially minimized at $\alpha_{d e t}=60^{\circ}$ (see Figure 5(b)), and complete elimination of sidelobes occurs at high NA detection $\left(\alpha_{\text {det }}=\right.$ $72^{\circ}$ ). This trend occurs because of the high lateral resolution of the detection system. For the proposed imaging system, the axial resolution is determined by the product of the individual excitation nanodot and the lateral PSF of the detection objective, $\mathrm{O}_{3}$. Overall, detection at large aperture angles provides information from individual excitation dots. Because the high NA illumination objective is practically helpful, we have also studied a different configuration of M-MESO system, in which we varied the illumination aperture angle $\left(\alpha_{i l l}=30^{\circ}, 60^{\circ}, 72^{\circ}\right)$ and fixed the detection aperture angle $\alpha_{\text {det }}$ at $45^{\circ}$, as shown in Figure 6. The nanodots are observed to elongate along the optical axis for large $\alpha_{i l l}$, thereby increasing the distance between two nearby nanodots. As, a result, the crosstalk from the neighboring nanodots is completely eliminated (see the intensity plots in Figure 6). This configuration is beneficial for artifact-free high resolution imaging. To elucidate the multilayer imaging capability of the
M-MESO system, we have detected nanodots at varying depths ( $\mathrm{z}$ layers). Figure 7 , shows the detection of target nanodot situated at depths of $540 \mathrm{~nm}$ and $1.56 \mu \mathrm{m}$, along with the detection of a central nanodot (at $z=0 \mathrm{~nm}$ ). The target nanodots are indicated by red arrows in the excitation PSF $\left(h_{\text {exc }}\right)$. Simultaneous detection from all nanodots can be obtained by using appropriate optical elements, such as, a diffraction grating in the detection path of the imaging system $^{19}$. Such a capability is useful for near-simultaneous indepth multilayer imaging of biological specimens.

The optical transfer function (OTF) of an imaging system provides bandwidth information. The OTF is obtained by the convolution of the excitation and detection OTFs. The OTFs for both the singleand multi- photon versions of the proposed imaging system are shown in Figure 8. The OTF peaks at zero frequency $\left(k_{x y}=0, k_{z}\right.$ $=0$ ). Missing bands were observed along the optical axis for single photon MESO, while the M-MESO imaging system shows continuously filled bands. The role of multiphoton excitation is to fill the missing bands in the three dimensional OTF. Gaps in the OTF indicate missing object spectrum information. Overall, better-filled bandwidth improves the efficiency of $3 \mathrm{D}$ image reconstruction because gaps in the OTF pose problems to the ill-posed image reconstruction process. Additionally, the two distinct sidelobes in the single photon MESO indicate substantial crosstalk that is missing in M-MESO, thereby illustrating the advantage of the M-MESO technique over its single photon variant.

Practical situations demand knowledge of the field distribution for both fixed and fluid samples. In fluid samples, the dipoles are free to rotate whereas the dipoles are static in fixed samples. As a result, the same field structure cannot be assumed for both cases, and the dipole orientation must be taken into account for field calculations. The interaction between the electric field and the molecular dipoles must also be considered. Scalar-diffraction theory has proven to be valuable for understanding the structure of the electric field of various optical components, such as lenses. However, the theory is not applicable for high numerical aperture (NA) objective lenses, which requires the tight focussing of light for fluorescence-based applications. Moreover, the theory lacks vectorial features of the electric field, such as, polarization, and it is unable to explain polarization dependent effects. This scenario requires the complete clasical theory of light, i.e, the vectorial-diffraction theory. Vectorial theory has been employed to quantify the electromagnetic field structure arising from both field polarization and dipole orientation for the proposed M-MESO imaging system. Figure 9 (top) shows the lateral (XY) field
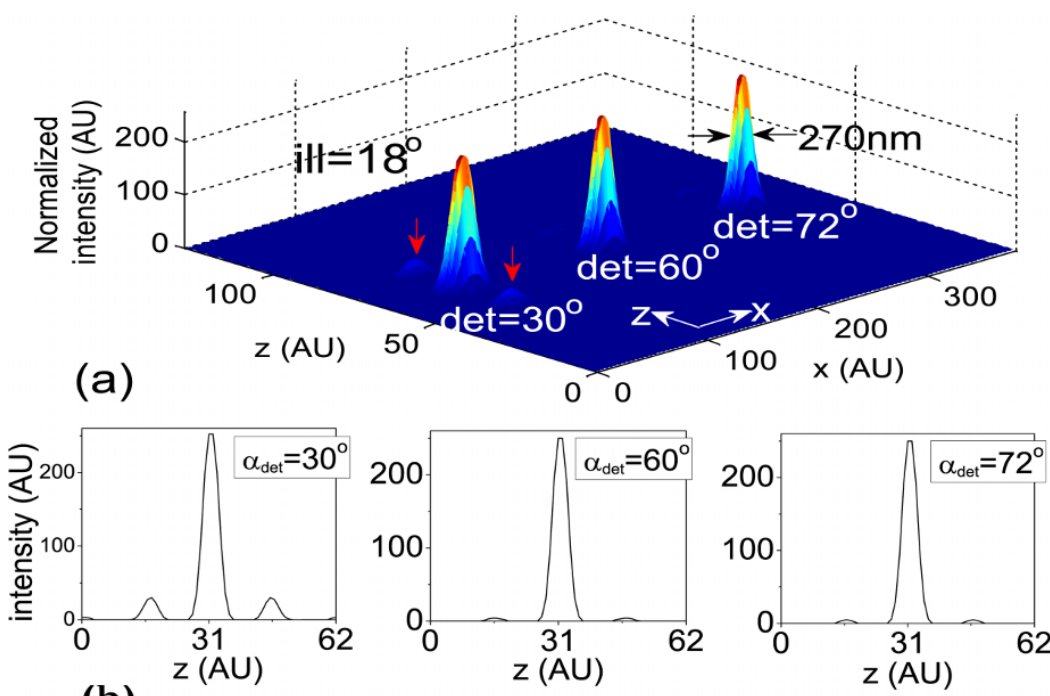

(b)

Figure $5 \mid$ (a) System PSF for varying detection aperture angle $\alpha_{d e t}=\left(30^{\circ}, 60^{\circ}, 72^{\circ}\right)$ and illumination aperture angle $\alpha_{i l l}=18^{\circ},(\mathrm{b})$ Corresponding intensity plots. 

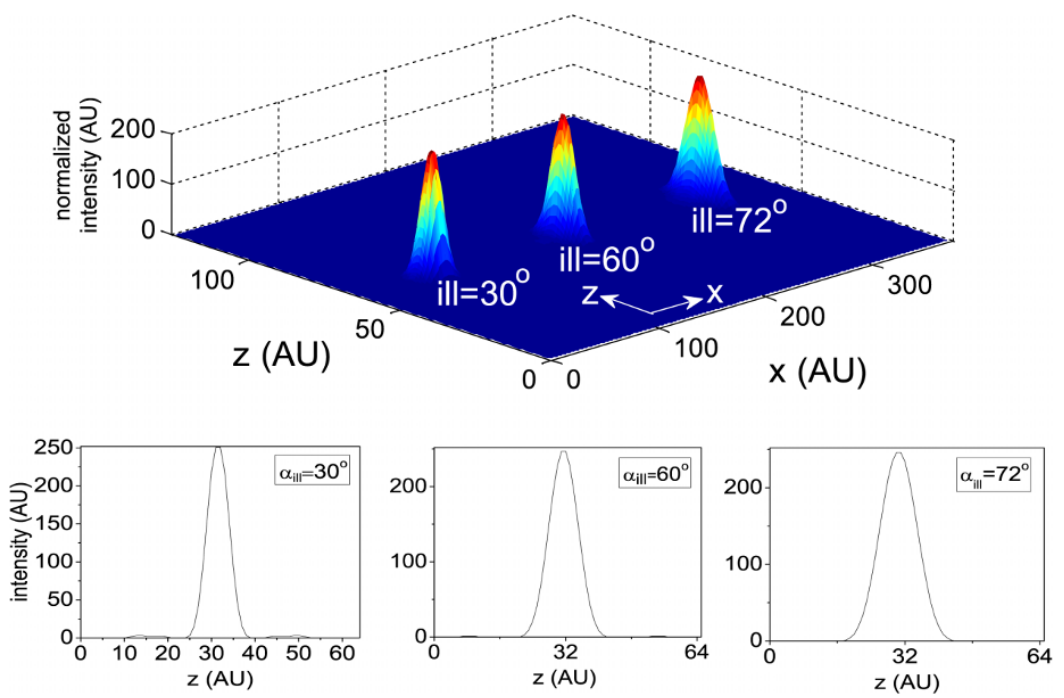

Figure $6 \mid$ (a) System PSF for varying illumination aperture angle $\alpha_{i l l}=\left(30^{\circ}, 60^{\circ}, 72^{\circ}\right)$ and fixed detection aperture angle $\alpha_{\text {det }}=45^{\circ}$, (b) Corresponding intensity plots.
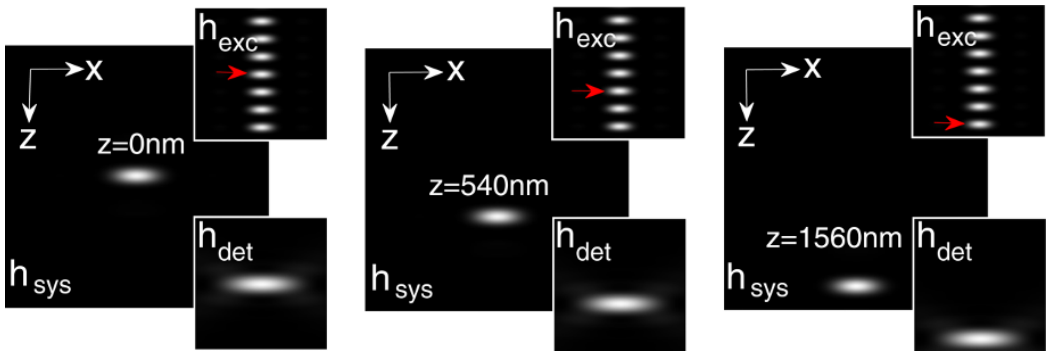

Figure $7 \mid$ Nanodot detection at varying z-layers: $0 \mathrm{~nm}, 540 \mathrm{~nm}$ and $1560 \mathrm{~nm}$. Red arrow indicates a target nanodot in the excitation PSF. Both the $x$ - and $z$ - axes extends from $-1.92 \mu m$ to $1.92 \mu m$.
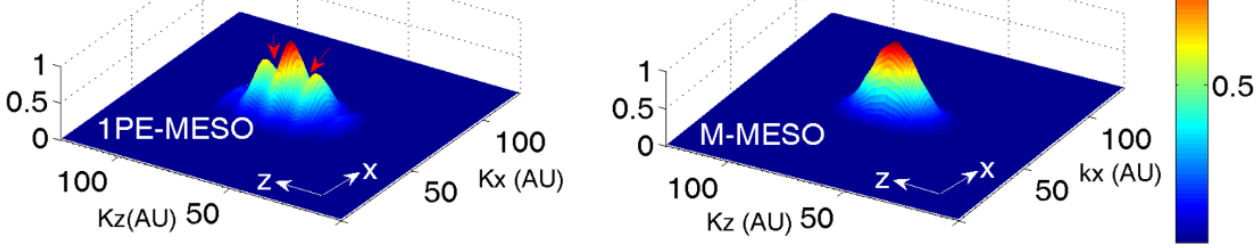

Figure 8 Optical transfer functions for both the single photon MESO and M-MESO imaging systems. Red arrows indicates missing bands in 3D OTF of single photon MESO system.

distribution for free-rotating dipoles illuminated by both randompolarized (RP) and linear-polarized (LP) light. The region of uniform excitation is large for LP light, but for RP light illumination, the uniformity is split into three prominent parts (see Figure 9, top-left). This result indicates that, LP light is beneficial for applications requiring uniform excitation. Figure 9 (top row) and (bottom row) show the field structure of a free rotating (representing fluidic sample) and $x$-oriented dipole (representing fixed sample), respectively. Irrespective of the polarization of the electric field, the field structure is significantly different and depend strongly on the dipole orientation, thereby eliciting the prominance of the field-dipole interaction. Understanding the effect of light polarization on the orientation of dipoles is essential for the realization of a precision super-resolution imaging system.

Finally, the proposed system $(M-M E S O)$ is compared with twophoton excitation $(2 P E)$ and two-photon excited $4 p i(2 P E-4 p i)$ imaging systems. Confocal detection perpendicular to the optical axis is employed for all cases. The illumination $\left(h_{i l l}\right)$, detection $\left(h_{\text {det }}\right)$, and the system PSF $\left(h_{\text {sys }}\right)$ are shown in Figure 10 for all imaging modalities. High resolution is evident in the $2 \mathrm{PE}$ system because of its confocal theta detection. Comparatively, the resolution is much better for $2 P E-4 p i$ and $M-M E S O$. However, the $2 P E-4 p i$ system can generate only one super-resolution dot, whereas, the proposed $M-M E S O$ system can generate several such high-resolution dots of dimension $\Delta z \approx 150 \mathrm{~nm}$. Additionally, the M-MESO system is capable of fluorescence detection from each individual nanodot without any cross-talks from neighboring nanodots.

\section{Discussion}

In principle, it is possible to further reduce the layer thickness with the use of a high NA objective. However, this alteration reduces the working distance thereby making it difficult to realize in practice. On 

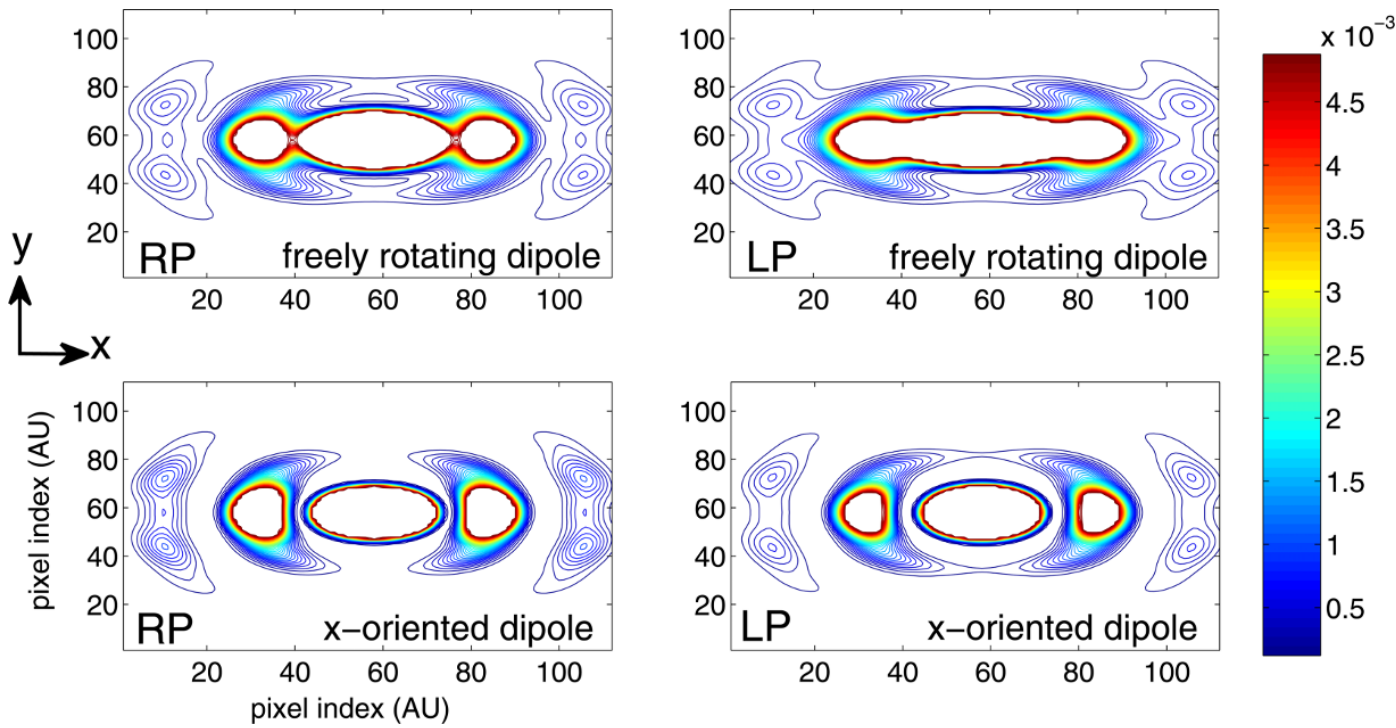

Figure 9 Field structure of four different combinations of field polarization and dipole orientation: (top) RP and LP field on freely rotating dipole, (bottom) RP and LP field on x-oriented fixed dipoles.

the other hand, it is also possible to increase the number of excitation dots by decreasing the NA of the excitation objective, but this variation enhances the cross-talks between the nearby nanodots, destroying the unique detection of fluorescence from each nanodot. Consequently, a balance must maintained for practically realizing the proposed imaging technique. Nonetheless, the parallel excitation of multiple specimen layers using nanodots is exciting. A spatial filter based multidot illumination, coupled with an orthogonal detection PSF, shows considerable tolerance for crosstalk-free fluorescence collection. This excitation technique is promising because the spatial filter pattern determines the shape and size of the system PSF. In addition, such filters enable diffraction-unlimited lateral resolution, sub-femto-liter volume excitation and the generation of Bessel-like beams ${ }^{28,29}$. As a result of the convenience and new possibilities associated with M-MESO, we anticipate that this technique may become a powerful tool for numerous applications in Biology, nanoscale imaging and optical engineering. These applications may include multiple-trap optical tweezers and multi-dot fluorescence correlation spectroscopy.

Scattering and anisotropy have a significant effect on the quality of the image and on multi-dimensional image reconstruction. These effects are minimized for two-photon imaging that uses, near infrared radiation (IR) for multiphoton excitation because the use of IR minimizes scattering ${ }^{22,23,24}$. The quadratic dependence on the excitation light intensity for two-photon excitation makes scattering less important because the excited fluorophore is spatially localized in the

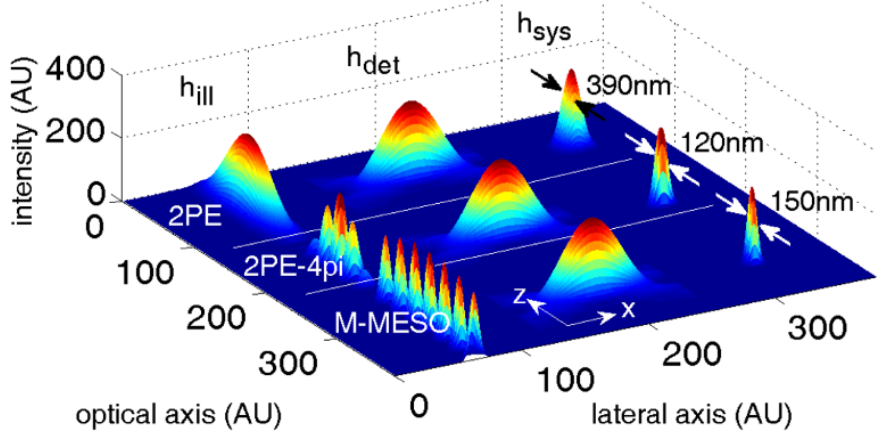

Figure $10 \mid$ Comparison of 2PE, 2PE-4pi and M-MESO PSF at an illumination and detection aperture angle of $45^{\circ}$. highest intensity regions of the beam. This mechanism, which is associated with two-photon excitation, leads to lower background and less photobleaching. In Rayleigh scattering, which is the most prominent scattering process for particles much smaller than the wavelength of light, the amount of scattered light scales inversely as the fourth power of the wavelength. Therefore, at an excitation wavelength of $910 \mathrm{~nm}$ (used for 2PE), scattering intensity is approximately 8 times less than for $488 \mathrm{~nm}$ (used for 1PE) excitation. This reduction in scattering increases the depth of penetration in thick biological specimens. Biological specimens are non-uniform and have variable indices of refraction, which causes multiple scattering of light in various directions. Specifically, in fluorescence imaging, the incident light is scattered to varying degrees before it reaches the focal plane. The resulting fluorescent light also undergoes similar scattering as it travels through the specimen toward the detector. Both of these scattering effects reduce the collected fluorescence signal, resulting in numerous image artifacts and hampering the image resolution. On the other hand, for particles a few microns in size, Mie scattering is dominant, and the scattering efficiency (defined in terms of the scattering cross-section and anisotropy) of the specimen determines the image resolution ${ }^{25}$. A decrease in the scattering efficiency implies a reduction in the number of multiple scattering events, resulting in increased signal collection by the detector and high image resolution. TPE is reported to have an image resolution that is two orders of magnitude higher than that under $1 \mathrm{PE}^{25}$.

\section{Methods}

M-MESO excitation is employed for the simultaneous multi-layer slicing of biological specimens. An efficient detection scheme is used to collect photons from the target nanodots to realize the complete imaging system (see Figure 1). We recognize confocal theta detection as the most suitable detection scheme for the proposed imaging system. Phase-matched light is structured by the spatial filter (imprinted on the objective lenses $O_{1}$ and $O_{2}$ ) and focused to the common geometrical focus of a $4 \pi$ geometry ${ }^{9,26,27}$ ( $4 \pi$-geometry is extensively used in fluorescence microscopy for axial super-resolution $\left.{ }^{9}\right)$. This focusing results in the formation of a series of bright nanodots because of interference along the optical axis. Aperture engineering on a single lens configuration with the proposed spatial filter produces a Bessel-like depth-offocus PSF $^{28,29}$. Other spatial filters are also proposed for high resolution imaging ${ }^{30,31,32}$. Fluorescence from these tiny nanodots is collected by the objective $\mathrm{O}_{3}$, which is positioned orthogonally to the optical axis (see, Fig 1). For the proposed study, the two-photon excitation and emission wavelengths are chosen to be $910 \mathrm{~nm}$ and $520 \mathrm{~nm}$ respectively. The excitation and emission/detection wavelength for the single photon variant are chosen to be $488 \mathrm{~nm}$ and $520 \mathrm{~nm}$, respectively. The 3D image consists of $128 \times 128$ pixels and 128 slices, with the geometrical focus located at $z=0$. A sampling rate (well above the Nyquist sampling) of about $30 \mathrm{~nm}$ is chosen along 
both the axial and lateral axes. To generate excitation nanodots, a transmission window of $\Delta \theta=\left(\alpha-\theta_{1}\right)=5^{\circ}$ is chosen for all cases (see Figure 1).

A schematic diagram of the proposed imaging system is shown in Figure 1.

In the Cartesian coordinate system, the $x$-, $y$ - and $z$-components of the electric field for randomly polarized light illumination are given by ${ }^{21},\left[E_{x}, E_{y}, E_{z}\right]=\left[-i A\left(I_{0}+I_{2}\right.\right.$ $\left.\cos (2 \phi)),-i A I_{2} \sin (2 \phi),-2 A I_{1} \sin (\phi)\right]$, where $A$ is the proportionality constant representing the amplitude of the incident electric field. Assuming substantial interaction between the incident electric field and the random-oriented dipoles, the excitation PSF of the M-MESO system is, $h_{i l l}(x, y, z)=$ $|\bar{E}(x, y, z)+\bar{E}(x, y,-z)|^{4}=\left[\left|\operatorname{Re} \tilde{I}_{0}\right|^{2}+2\left|\operatorname{Re} \tilde{I}_{1}\right|^{2}+\left|\operatorname{Re} \tilde{I}_{2}\right|^{2}\right]^{2}$, where $\tilde{I}_{0,1,2}=$ $\int_{\theta=0}^{\alpha_{\text {ill }}} B(*) G_{0,1,2}(*) \cos ^{1 / 2} \theta e^{i\left(\frac{u \cos \theta}{\sin ^{\alpha} \alpha i l l}\right)} d \theta$ are modified diffraction integrals with, $\left[G_{0}(*), G_{1}(*), G_{2}(*)\right]=\left[\sin \theta(1+\cos \theta) J_{0}(w), \sin ^{2} \theta J_{1}(w), \sin \theta(1-\cos \theta) J_{2}(w)\right]$, where, $w=(v \sin \theta / \sin \alpha)$. The function, $B\left({ }^{*}\right)=H\left[\theta-\theta_{1}\right]-H[\theta-\alpha]$ represents the spatial filter with cutoff angle $\theta_{1}$, for which the transmission window is $\Delta \theta=5^{\circ} ; H$ is the Heaviside function. $u=\frac{2 \pi}{\lambda} z \sin ^{2} \alpha$ and $v=\frac{2 \pi}{\lambda} \sqrt{x^{2}+y^{2}} \sin \alpha$ are the longitudinal and transverse optical coordinates, respectively ${ }^{21}$, and $\alpha_{i l}$ is the illumination semiaperture angle. The numerical aperture of the objective lens in air is defined as, $\sin \alpha$, where $\alpha$ is the semi-aperture angle. For single photon MESO, the illumination PSF is, $h_{\text {ill }}=\left|\operatorname{Re} \tilde{I}_{0}\right|^{2}+2\left|\operatorname{Re} \tilde{I}_{1}\right|^{2}+\left|\operatorname{Re} \tilde{I}_{2}\right|^{2}$. We employ a confocal theta detection scheme, in which the detection is carried out in the orthogonal plane, as shown in Figure 1. The orthogonal plane of detection is represented by the following transformation: $\left(x^{\prime}=\right.$ $-z, y^{\prime}=y, z^{\prime}=x$ ). This detection scheme has the advantage of high resolution at long working distance. The isotropic emission model is assumed with randomly polarized light excitation. The corresponding detection electric field is, $h_{\text {det }}\left(x^{\prime}, y^{\prime}, z^{\prime}\right)=$ $\left|\bar{E}_{x^{\prime}}\right|^{2}+\left|\bar{E}_{y^{\prime}}\right|^{2}+\left|\bar{E}_{z^{\prime}}\right|^{2}$, where the integration $I_{0,1,2}=\int_{\theta=0}^{x_{d x t}}(\cdots) d \theta$ on the aperture-free objective $\left(\mathrm{O}_{3}\right)$ is carried over the detection semi-aperture angle $\alpha_{\text {det }}$. Overall, the system PSF of the proposed imaging system is given by, $h_{s y s}(x, y, z)=h_{i l l}(x, y, z) \times$ $h_{\text {det }}(-z, y, x)$.

The incident electric field gives rise to an oscillating dipole $(\vec{p})$, which in turn produces a far-field electric field given by, $\vec{E}=-\vec{r} \times(\vec{r} \times \vec{p})$. Assuming paraxial optics and using the two-dimensional (2D) Fourier transform, one can obtain explicit expressions for the calculation of the time-averaged electric energy density, following the approach of Sheppard et a ${ }^{33}$. Here, the field is calculated for four different cases. Initially, the RP light on a freely rotating dipole is considered, for which the field distribution is, $h_{R P \text { free rot }}=\left[\left|\operatorname{Re} \tilde{I}_{0}\right|^{2}+2\left|\operatorname{Re} \tilde{I}_{1}\right|^{2}+\left|\operatorname{Re} \tilde{I}_{2}\right|^{2}\right]^{2}$. For $x$-oriented dipoles, the field distribution becomes $h_{R P, x-\text { orient }}=\left[\left|R e \tilde{I}_{0}\right|^{2}+2\left|R e \tilde{I}_{1}\right|\left|R e \tilde{I}_{2}\right| \cos (2 \phi)+\right.$ $\left.\left|R e \tilde{I}_{2}\right|^{2}\right]^{2}$. Considering LP light on a randomly oriented dipole, the field distribution is given by, $h_{L P \text { free-rot }}=\left[\left|R e \tilde{I}_{0}\right|^{2}+4\left|R e \tilde{I}_{1}\right|^{2} \cos ^{2} \phi+\left|R e \tilde{I}_{0}\right|^{2} \cos (2 \phi)+\left|R e \tilde{I}_{2}\right|^{2}\right]^{2}$, and the field distribution becomes, $h_{L P, x-\text { orient }}=\left[\left|R e \tilde{I}_{0}\right|^{2}+2\left|\operatorname{Re} \tilde{I}_{0}\right|\left|\operatorname{Re} \tilde{I}_{2}\right| \cos ^{2} \phi+\right.$ $\left.\left|R e \tilde{I}_{2}\right|^{2} \cos 2 \phi\right]^{2}$ for a fixed dipole orientation along $\mathrm{x}$.

In the case of fast imaging, to scan $M$ slices of an $N \times N$ image, a laser scanning system requires $M \times N^{2}$ scan-points and a total time of $\left(M \times N^{2}\right) \Delta t$. The generation of an excitation PSF with $M$ axial dots, allows in-principle the data acquisition rate to be increased by a factor of $M$, thereby reducing the total acquisition time by a factor of $M$ $\Delta t$. The proposed technique can be useful for fast imaging of biological specimens with reduced background and photobleaching effects.

1. Voie, A. H., Burns, D. H. and \& Spelman, F. A. Orthogonal-plane fluorescence optical sectioning: three-dimensional imaging of macroscopic biological specimens. J. Microsc. 170, 229-236 (1993).

2. Brakenhoff, G. J., Blom, P. \& Barends, P. Confocal scanning light microscopy with high aperture immersion lenses. J. Micros. 117, 219-232 (1979).

3. Diaspro, A., Chirico, G. \& Collini, M. Two-photon fluorescence excitation and related techniques in biological microscopy. Quart. Rev. Biophys. 38, 1-97 (2005).

4. Denk, W., Strickler, J. H. \& Webb, W. W. Two-photon laser scanning fluorescence microscopy. Science 248, 73-76 (1990).

5. Diaspro, A. et al. Multi-photon excitation microscopy. Biomed. Engg. 5, 36-50 (2006).

6. Mazza, D et al. A new FRAP/FRAPa method for three-dimentsional diffusion measurements based on multiphoton excitation microscopy. Biophys. J. 95, 34573469 (2008).

7. Huisken, J., Swoger, J., Del Bene, F., Wittbrodt, J. \& Stelzer, E. H. K. Optical sectioning deep inside live embryos by selective plane illumination microscopy. Science 305, 1007-1009 (2004)

8. Planchon, T. A. et al. Rapid three-dimensional isotropic imaging of living cells using Bessel beam plane illumination. Nature Methods 8, 417-423 (2011).

9. Hell, S. W., Schmidt, R. \& Egner, A. Diffraction-unlimited three-dimensional optical nanoscopy with opposing lenses. Nat. Photon. 3, 381-387 (2009).

10. Folling, J. et al. Fluorescence Nanoscopy with Optical Sectioning by Two-Photon Induced Molecular Switching using Continuous-Wave Lasers. Chem. Phys. Chem. 9, 321-326 (2008).

11. Song, L. L., Hennink, E. J., Song, L. L., Hennink, E. J., Young, I. T. \& Tanke, H. J. Photobleaching kinetics of fluorescein in quantitative fluorescence microscopy. Biophys. J. 68, 2588-2600 (1995).

12. Esposito, A. et al. Quantitative fluorescence microscopy techniques. Meth. Mol. Biol. 586, 117-142 (2009).
13. Patterson, G. H. \& Piston, D. W. Photobleaching in two-photon excitation microscopy. Biophys J. 78, 2159-2162 (2000).

14. Mondal, P. P., Gilbert, R. J. \& So, P. T. C. Photobleaching reduced fluorescence correlation spectroscopy, Appl. Phys. Lett. 97, 103704 (2010).

15. Mondal, P. P. \& Diaspro, A. Reduction of higher-order photobleaching in twophoton excitation microscopy. Phys. Rev. E 75, 061904 (2007).

16. Mondal, P. P. Multiple excitation nano-spot generation and confocal detection for far-field microscopy. Nanoscale 2, 381-384 (2010).

17. Mondal, P. P. Chain of excitation spot generation by aperture engineering. Jl. Appl. Phys. 107, 086101 (2010).

18. Stelzer, E. H. K. \& Lindek, S. Fundamental reduction of the observation volume in far-field light microscopy by detection orthogonal to the illumination axis: confocal theta microscopy. Opt. Comm. 111, 536-547 (1994).

19. Dalgarno, P. A. et al. Multiplane imaging and three dimensional nanoscale particle tracking in biological microscopy. Opt. Exp. 18, 877-884 (2010).

20. Juette, M. F. \& Bewersdorf, J. Three-Dimensional Tracking of Single Fluorescent Particles with Submillisecond Temporal Resolution. Nano Lett. 10, 46574663 (2010).

21. Boivin, A. and Wolf, E. Electromagnetic field in the neighborhood of the focus of a coherent beam. Phys. Rev. 138, B1561-B1565 (1965).

22. Truong, T. V., Supatto, W., Koos, D. S., Choi, J. M. \& Fraser, S. E. Deep and fast live imaging with two-photon scanned light-sheet microscopy, Nature Methods 8 , 757-760 (2011).

23. Keller, P. J. et al. Fast, high-contrast imaging of animal development with scanned light sheet-based structured-illumination microscopy, Nature Methods 7, 637$642(2010)$.

24. Reynaud, E. G., Krzic, U., Greger, K. \& Stelzer, E. H. K. Light sheet-based fluorescence microscopy: more dimensions, more photons, and less photodamage, HFSP Jl. 2, 266-275 (2008)

25. Gu, M., Gan, X., Kisteman, A. \& Xu, M. G. Comparison of penetration depth between two-photon excitation and single-photon excitation in imaging through turbid tissue media, Appl. Phys. Lett. 77, 1551-1553 (2000).

26. Cremer, C. \& Cremer, T. Considerations on a laser-scanning-microscope with high resolution and depth of field. Microsc. Acta 81, 31-44 (1978).

27. Hell, S. W., Stelzer, E. H. K., Lindek, S. \& Cremer, C. Confocal microscopy with an increased detection aperture: type B 4Pi-confocal microscopy. Opt. Lett. 19, 222224 (1994).

28. Mondal, P. P. \& Diaspro, A. Lateral resolution improvement in two-photon excitation microscopy by aperture engineering. Opt. Comm. 281, 1855-1859 (2008).

29. Mondal, P. P., Mandal, S. \& Diaspro A. Dynamic point spread function for single and multiphoton fluorescence microscopy. Rev. Sci. Instrum. 81, 046103 (2010).

30. Toraldo di Francia, G. Super-gain antennas and optical resolving power. Nuovo Cimento 9, 426-435 (1952).

31. Gustafsson, M. G. L. Nonlinear structured-illumination microscopy: Wide-field fluorescence imaging with theoretically unlimited resolution. Proc. Nat. Acad. Sci. USA 102, 13081-13086 (2005).

32. Wang, H., Shi, L., Yanchuk, B. L., Sheppard, C. \& Chong, T. C. Creation of a needle of longitudinally polarized light in vacuum using binary optics, Nat. Phot. 2, 501505 (2008).

33. Sheppard, C. J. R. and Torok, P. An electromagnetic theory of imaging in fluorescence microscopy and imaging in polarization fluorescence microscopy, Bioimaging 5, 205-218 (1997).

\section{Acknowledgements}

PPM would like to acknowledge BRNS (DAE), Department of Science and Technology and the parent institute (Indian Institute of Science) for financially supporting the research work at the Nanobioimaging Laboratory, Department of Instrumentation and Applied Physics, Indian Institute of Science. AD would like to acknowledge MIUR PRIN 2008JZ4MLB.

\section{Author contrubutions}

PPM and AD conceived and designed the study. PPM and AD performed the theoretical studies, and the computational studies were carried out by PPM. PPM and AD analyzed the data. PPM wrote the manuscript in consultation with $\mathrm{AD}$.

\section{Additional information}

Competing financial interests: The authors declare no competing financial interests.

License: This work is licensed under a Creative Commons

Attribution-NonCommercial-ShareAlike 3.0 Unported License. To view a copy of this license, visit http://creativecommons.org/licenses/by-nc-sa/3.0/

How to cite this article: Mondal, P.P. \& Diaspro, A. Simultaneous multilayer scanning and detection for multiphoton fluorescence microscopy. Sci. Rep. 1, 149; DOI:10.1038/ srep00149 (2011) 